\title{
The relationship between mortality and two indicators of morbidity
}

\author{
MARY E. BRENNAN AND PHILIP H. CLARE \\ From the West Midlands Regional Health Authority, Birmingham
}

SUMmaRY The Resource Allocation Working Party concluded that standardised mortality ratios are the best available indicators of geographical variations in morbidity. In this paper we give the results of a statistical analysis of the relationship between mortality and two indicators of morbidity, obtained from the 1971 census, for three age groups. The level of aggregation in the data is comparable with that at district or area level. Strong linear relationships are obtained, suggesting that it is reasonable to use mortality data in the RAWP formulae in applications at area or district level. However, this method of resource allocation should not be used in isolation from planning. A possible solution which reduces conflict between the two approaches is to incorporat巴 mortality data in the planning indicators used to establish relative need and, in addition, to take due account of established patterns of service and local circumstances.

The report of the Resource Allocation Working Party (RAWP) in $1976^{1}$ contained recommendations which will have considerable implications for the supply of health care services in this country. The pattern of the distribution of financial resources was intended to be 'responsive objectively, equitably and efficiently to relative need', and the method of distribution proposed by RAWP, and subsequently implemented, is certainly a great improvement on the previous method of incrementation which tended to perpetuate geographical inequalities in access to health care.

In this paper we present the results of a statistical analysis of the relationship between mortality and two measures of morbidity for specific age groups obtained from the 1971 census. Various commentators have, between them, discussed the conditions under which the use of the standardised mortality ratio (SMR) for RAWP purposes would be acceptable. We have focused on two of these conditions to see if our data suggest whether they are in fact likely to be fulfilled. The two requirements are: the existence of a strong relationship between mortality and morbidity at low levels of aggregation (that is, below regional level); and the need for a linear relationship. We have confined our research to all-condition mortality.

\section{Method}

Data from the 1971 census were collected for the following population groups in England: (a) each county borough except the City of London (111 data points); (b) each county aggregate of metropolitan boroughs and urban districts (45 data points); and (c) each county aggregate of rural districts (45 data points).

Thus the level of aggregation we investigated was lower than county (as constituted before the 1974 local government reorganisation) and, therefore, lower than AHA. Morbidity information obtained from the census was expressed in terms of two indicators:

(i) Short- and medium-term morbidity was estimated by the numbers in the population assessing themselves as 'sick and therefore unemployed'.

(ii) Long-term morbidity was estimated by the numbers assessing themselves as 'permanently sick and therefore not seeking employment'.

The assessments were made by people who had of been without a job in the week ended 24 April 1971. It follows that morbidity data for children (0-15 age group) were not available from the census. The total number of deaths (male and female combined) for 
the age groups $15-44,45-64$ and $65+$ were obtained from the Office of Population Censuses and Surveys (OPCS) (form SD25) for each county in each population group. Both the mortality and the morbidity data were grouped into several age bands in the source reports but the three age groups quoted above were the only ones common to both sets of data. The numbers of deaths, and the numbers of sick and permanently sick, were converted to rates by dividing by the appropriate population number in each age group.

Correlations between the mortality rate and the sick and permanently sick for each age group were calculated using least squares linear regression.

A difficulty was encountered with the $65+$ age group. We suspected that the respondents to the two morbidity questions in the 1971 census were likely to be only a proportion of the $65+$ population, because the questions were about sickness in the context of employment. In other words, we doubted that the information was representative of that age group. When we examined the rates of sick and permanently sick in each age group, the findings supported our view: the rates for those aged 65 and over were much lower than the rates for the other age groups, which is contrary to what would be expected for the elderly.

We therefore postulated that the real level of morbidity in those aged 65 and over would be strongly correlated with the level of morbidity in the 45-64 age group, and on this assumption we examined the relationship between the mortality of those aged 65 and over and the morbidity of the 45-64 age group.

Regression was used to explore not just the strength of the relationship between the two rates but also the nature of that relationship. A visual inspection of the data plotted graphically suggested a general model of the following form for each age group:

$$
{ }^{*} m_{k}=a+b_{1} M_{k}+b_{2} M_{k}^{2}+e_{k}
$$

Although the relationship is non-linear, we used linear regression, treating the square of morbidity term as an additional variable in a linear equation. This approach is adequate for testing whether a non-linear model is better than a linear one, but it is no good for determining the coefficients in a polynomial. Our justification is that the results we obtained indicated that there was no point in proceeding to the estimation of coefficients.

\footnotetext{
- $\quad m_{k}=$ mortality rate of the $k^{\text {th }}$ location in the age group $\mathbf{M}_{\mathbf{k}}=$ morbidity rate of the $\mathbf{k}^{\text {th }}$ location in the age group $\mathrm{e}_{\mathbf{k}}=$ error between predicted mortality rate and actual mortality rate for the $k$ location; $e_{k}$ assumed to be distributed normally about zero.

$a, b_{1}, b_{2}=$ constants, to be determined by regression, which minimise the sum of the $e^{2} k$.
}

We treated mortality as the dependent variable throughout the analysis; in other words we regarded deaths as arising from ill health. It should be noted that we did not use the SMR for each geographical area; this is a summary statistic for deaths in all age groups, whereas our data excluded children and therefore prevented us from using SMRs. Instead, we examined mortality and morbidity in each of three age groups separately.

\section{Results}

Correlation coefficients obtained from linear regression, showing the association between mortality and the two indicators of morbidity, are shown in Tables 1 and 2 overleaf.

In the 15-44 age group, there is a very strong association between mortality and 'sick and out of employment' for both the total sample and the three separate population groups. However, for the 'permanently sick' and mortality relationship, our results show that it is only significant at the $95 \%$ level for each separate population group, although in the total sample the relationship is significant at the $99 \%$ level. Both measures of morbidity are highly correlated with mortality for the 45-64 age group, for the three separate population groups, and the total sample.

As mentioned earlier, for the $65+$ age group we analysed the relationship between mortality rates for that age group with morbidity for those aged 45-64. It is possible to imagine a causal relationship at work here for conditions with a prolonged history, particularly in the case of the permanently sick. Our results show strong relationships between mortality and both measures of morbidity. The conclusion is that, for most of the cases we have studied, there is a strong relationship between mortality rates and morbidity rates as measured by the indicators used in the 1971 census. None of the relationships we have investigated had more than a $5 \%$ chance of occurring randomly, and all except three results had less than a $1 \%$ chance of occuring randomly.

Using the regression model described above, we explored the nature of the relationship between mortality rates and morbidity rates. We found that, without exception, a linear equation was the most satisfactory fit to the data, that is, the square of the morbidity term did not significantly improve the correlation. For a visual illustration of the type of relationship, see the Figure on page 137, which shows a graph of the most highly correlated data.

Despite these positive results, it is evident from the magnitude of the derived correlation coefficients that the total variation in mortality rates is only partially explained by the total variation in morbidity rates. The explanatory power ranges from $5 \%$ to $54 \%$. 


\section{Discussion}

The incorporation of SMRs in the RAWP formulae has a significant impact on the calculated revenue targets. This can be illustrated with reference to

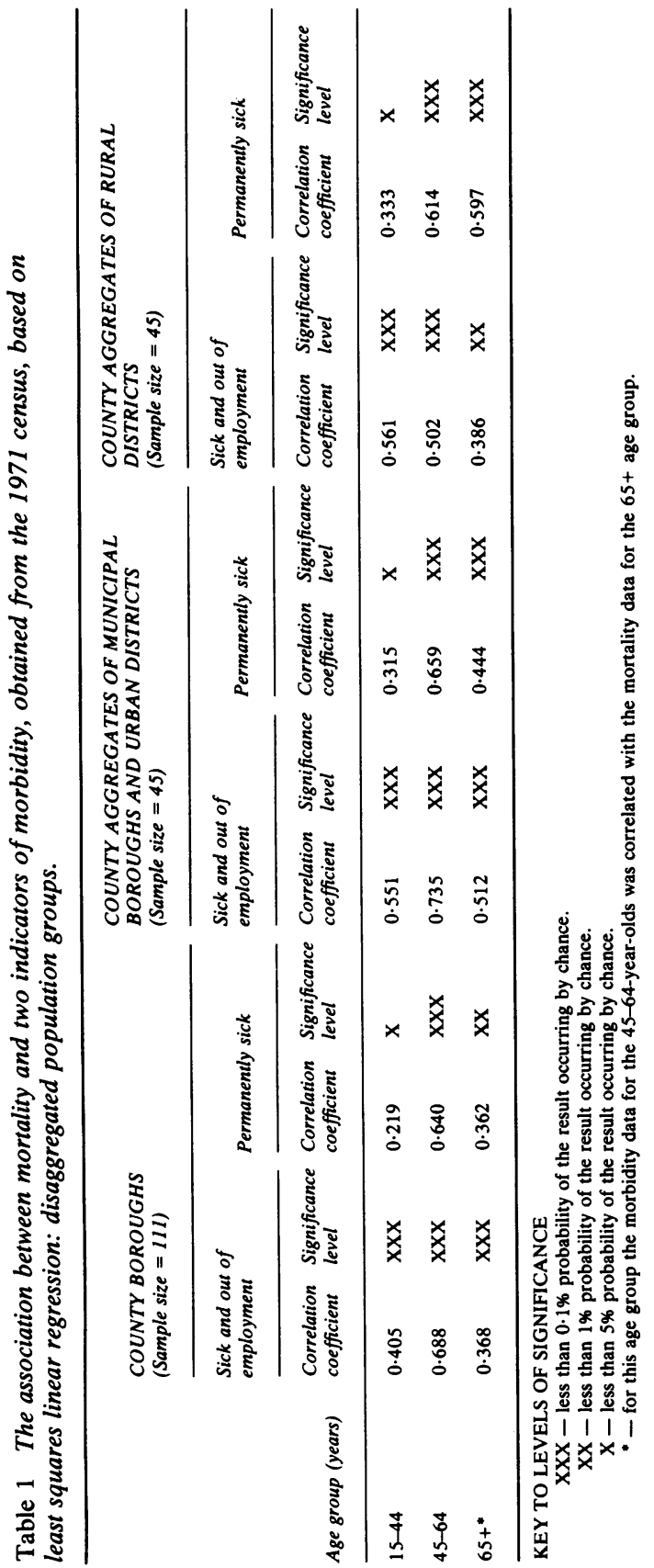

analysis done for the West Midlands RHA's Capital and Revenue Targets (CART) Working Party by its Technical Support Group. ${ }^{2}$ Revenue targets for each of the 11 AHAs in the region were calculated with and without the use of SMRs, and the percentage

Table 2 The association between mortality and two indicators of morbidity, based on least squares linear regression, for the total sample of all population groups

\begin{tabular}{|c|c|c|c|c|}
\hline \multirow[b]{3}{*}{ Age group (years) } & \multicolumn{4}{|c|}{$\begin{array}{l}\text { TOTAL SAMPLEOFALL POPULATIONGROUPS } \\
(\text { Sample size }=201)\end{array}$} \\
\hline & \multicolumn{2}{|c|}{$\begin{array}{l}\text { Sick and out of } \\
\text { employment }\end{array}$} & \multicolumn{2}{|c|}{ Permanently sick } \\
\hline & $\begin{array}{l}\text { Correlation } \\
\text { coefficient }\end{array}$ & $\begin{array}{l}\text { Significance } \\
\text { level }\end{array}$ & $\begin{array}{l}\text { Correlation } \\
\text { coefficient }\end{array}$ & $\begin{array}{l}\text { Significance } \\
\text { level }\end{array}$ \\
\hline $15-44$ & 0.444 & $\mathbf{X X X}$ & 0.203 & $\mathbf{X X}$ \\
\hline $45-64$ & 0.758 & $\mathbf{X X X}$ & 0.570 & $\mathbf{X X X}$ \\
\hline $65+$ & 0.435 & $\mathbf{X X X}$ & 0.370 & $\mathbf{X X X}$ \\
\hline
\end{tabular}

See Table 1 for key to significance levels.

change in the target, but not, of course the actual allocation, was noted for each AHA. The changes iv varied from $-3.7 \%$ to $+5.9 \%$ with a mean absolut $\vec{\omega}$ variation of $2 \cdot 7 \%$. The financial changes ranged from $\vec{A}$ $-£ 1.86 \mathrm{~m}$ to $+£ 2.34 \mathrm{~m}$. In view of variations of th\& magnitude, it is not surprising that the RAWP's use of SMR has been widely challenged, but it is als 8 clear that a recommendation with such importan 0 financial implications should be based securely o $\overrightarrow{0}$ supporting evidence.

RAWP produced some evidence in support of the use of SMR as a morbidity indicator. A comparison of age- and sex-standardised mortality rates with similarly adjusted morbidity-related data at a regional level of aggregation showed 'significant $\stackrel{\odot}{\varnothing}$ positive correlations'. The morbidity indicators used were sickness benefit statistics and self-reported acute and chronic illness reported in the General Household Survey. It was as a result of these studies that RAWP recommended the use of SMRs.

The first issue to which this research was directed was the contention that the relationship between? mortality and morbidity, which has been observed at regional levels of aggregation, is probably non-existent at lower levels of aggregation, that is, at $O$ area or district level.

Forster $^{3}$ examined the rank correlation between 을 mortality, directly standardised for age and sex, and $D$ similarly standardised morbidity rates from the General Household Survey, for the 10 standard statistical regions for 1972 and 1973 combined. He found a significant correlation between mortality and $\tilde{O}$ chronic sickness, but the correlation between $\underset{\omega}{N}$ mortality and absence from work or school due to injury or illness in males, though positive, was not 
significant. He therefore concluded that it seems reasonable to doubt whether standardised mortality ratio accurately measures relative need for health care'. However, this finding was probably related to the size of the sample, which was much smaller than in this study.

Snaith ${ }^{4}$ made a similar point suggesting that at lower levels of aggregation than region the inadequacy of SMR would become more marked, while Ferrer $e^{t} a^{5}$ stated that 'the relationship has not been conclusively demonstrated at regional level, but at lower levels of aggregation for areas and districts it is more doubtful if it exists'.

Our results do not support this view. We analysed data appertaining to geographical units smaller than the county, and all the relationships we studied were statistically significant at the $95 \%$ level and, in many cases, at the $99 \%$ and the $99.9 \%$ levels. In the absence of evidence to the contrary, we therefore concluded that it is legitimate to use age-specific mortality data in the RAWP formulae in applications at area or district level.

Regarding the second assumption made by RAWP and questioned by Ferrer $e t$ al, ${ }^{5}$ that the relationship between mortality and morbidity is a linear one, our results provide some support for it, because non-linearities in the relationship were found to be non-significant statistically. On the other hand, it could be argued that the degree of correlation found in our data, though statistically significant, was not sufficiently large to enable any non-linearities to be detected, should they exist. It would be necessary in any future work on non-linearities to work with more highly correlated data, if these could be found. Despite these significant correlations there is a lot of variation from one location to another, so that the constancy of the relationship does not appear to be substantiated. Undoubtedly this is due partly to the fact that our morbidity indicators represent different proportions of the total morbidity in different places, and partly to a real variation in the ratio of mortality to total morbidity. This study does not enable us to distinguish these two effects, and further work using differing morbidity indicators is required.

The two indicators of morbidity used in this study are only a partial measure of the total morbidity existing in any population. The first indicator, 'sick and therefore unemployed', is a measure only of morbidity sufficiently serious to restrict normal activities to such an extent that the person is temporarily unemployable. There are many types of illness which do not prevent attendance at work, but which present a significant demand on health services. Another implication is that the indicator, because it covers activity-restricting illness in people who would otherwise be employed, does not cover illness in those who would not, in any case, be employed. From these deficiencies it follows that the ratio of this morbidity indicator to total morbidity will vary geographically.

The long-term morbidity indicator, 'permanently sick', is also defined in terms of rendering a person unemployed, and it is therefore subject to the same criticisms as the short-term indicator. In particular,

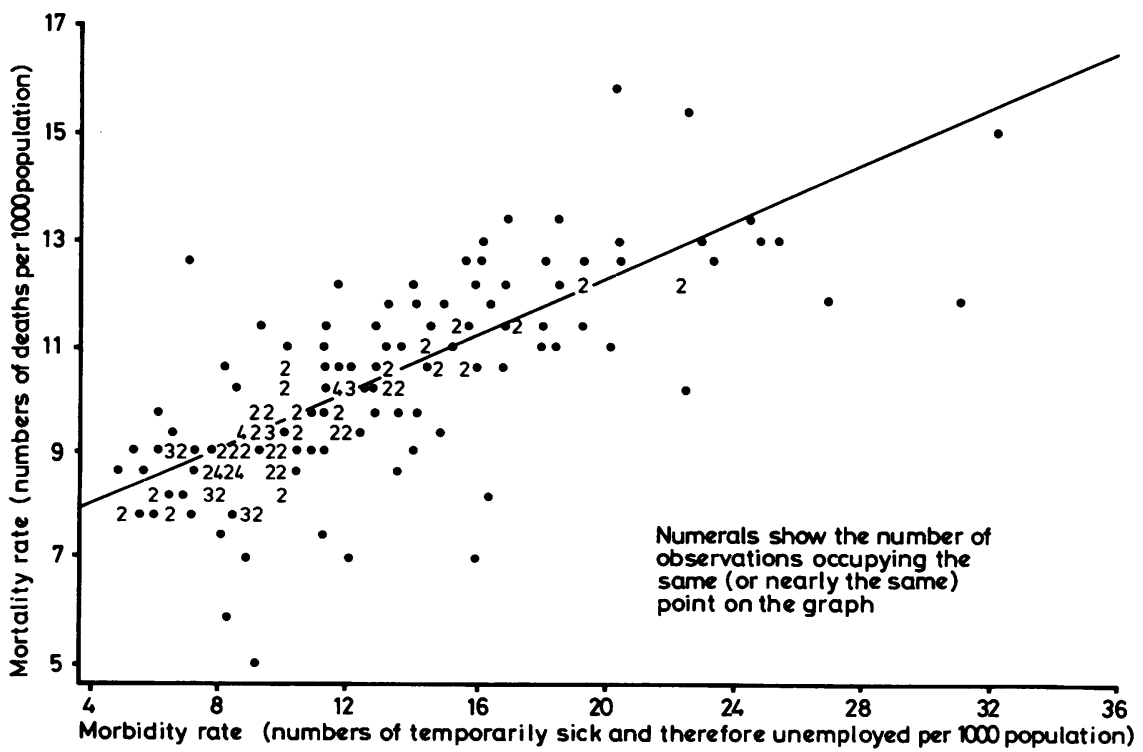

Figure Relationship between mortality and the short-term morbidity indicator for the 45-64 age group (total sample). 
we rejected the short- and the long-term morbidity data for the over-65s because it was clearly unrepresentative of illness in that age group. It is also likely that for the 15-44 age group the 'permanently sick' indicator is not a good cross-sectional measure of morbidity, because it could be expected that those people who were permanently sick in this age group would be suffering from a small group of particular conditions which would not be representative of the general level of morbidity in the age group.

We would, however, expect the 'permanently sick' in the age group 45-64 to be suffering from a wider spectrum of conditions than those in the 15-44 age group, and we would be more inclined to accept it as a meaningful measure of morbidity.

Despite the above criticism of these two indicators, we believe that they are a measure of morbidity at the severe or urgent end of its spectrum, leading to disability of a kind associated with serious social or economic dislocation for the individual.

Unfortunately, the poor quality of the morbidity data for the over-65s has prevented us from examining the relationship between mortality and morbidity for this age group. Therefore, this analysis justifies the use of mortality only as a proxy measure for morbidity in the two younger specified age groups, although if our assumption is correct, that morbidity of the $45-64 \mathrm{~s}$ is strongly correlated with that of the over- $65 \mathrm{~s}$, our conclusions will apply to the over-65s also. A strong positive correlation has been demonstrated between indicators of socioeconomic deprivation and age-specific mortality and morbidity in most age groups, ${ }^{678}$ which tends to support the contention that morbidity is positively correlated with mortality.

The starting point for this study was the RAWP formulae for revenue and capital allocation. We have shown that morbidity indicators are correlated with age-specific mortality data in administrative areas which are the size of National Health Service areas or districts, and that mortality data can therefore be used in any formula which defines targets for areas. It is our contention, however, that the allocation of capital and revenue should be determined by planning priorities rather than distance from RAWP targets. Any inherent conflict between these two approaches can be reduced by incorporating mortality data in the planning indicators used to establish relative need while, in addition, taking due account of established patterns of service and local circumstances. The latter requirement is most clearly relevant in the inner city areas, whether or not they are deprived in RAWP terms. ${ }^{9}$ We do not accept that equity should be ignored, but destructive dislocation of existing services will occur if RAWP targets are considered in isolation from planning.

We thank Mr. John Kelsall, Operational Research Unit, for collecting the data and undertaking the analyses. The views expressed in the paper are not necessarily those of the RHA. We thank Mrs. P. Mullen and Mr. I. Nicholls for helpful comments.

Reprints from Dr. Mary E. Brennan, West Midlands Regional Health Authority, Arthur Thomson House, 146, Hagley Road, Birmingham B16 9PA.

\section{References}

${ }^{1}$ Department of Health and Social Security. Sharing Resources for Health in England. London: HMSQ 1976.

${ }^{2}$ West Midlands Regional Health Authority. Capital an Revenue Target Working Party. Interim Report of the Technical Subgroup. Birmingham: West Midlang $\vec{E}$ RHA, 1977: 16.

${ }^{3}$ Forster DP. Mortality, morbidity and resource allocation. Lancet 1977; 1: 997.

${ }^{4}$ Snaith AH. Subregional resource allocations in the National Health Service. J Epidemiol Community Health 1978; 32: 16-21.

${ }^{5}$ Ferrer HP, Moore A, Stevens GC. The use of mortality data in the report of the Resource Allocation Working Party. Public Health 1977; 91: 289-95.

${ }^{6}$ British Medical Journal. Editorial: Children who die through social disadvantage. Br Med J 1976; ii: 962.

${ }^{7}$ Brennan M, Lancashire R. Association of childhood mortality with housing status and unemployment. $J$ Epidemiol Community Health 1978; 32: 28-33.

${ }^{8}$ Central Statistical Office. Social class: commentary. Social Trends. London: HMSO, 1975.

- Jones, Sir Frances Avery. National Health Service, RAWP, the Royal Commission and the inner cities. Lancet 1979; 2: 372. 